\title{
ETIOLOGY AND PATTERN OF DRUG INDUCED LIVER INJURY IN A TERTIARY GARE HOSPITAL OF NEPAL
}

\author{
K.C. A, Jha S, Thapa S
}

\begin{abstract}
Drug induced liver injury (DILI) is one of the common cause of liver toxicity. Most of the drugs used today are hepatotoxic. DILI accounts for approximately one-half of the cases of acute liver failure and mimics all forms of acute and chronic liver disease. It is the single most common adverse drug reaction leading to a halt in the development of new medication by pharmaceutical company, failure of new drug to obtain regulatory approval, and withdrawal or restriction of existing drug from the market. The aim of this study is to evaluate common causes and patterns of DILI in our setting. Twenty-seven patients were enrolled in the study. Antitubercular drugs were most common cause of DILI, accounting for $48.2 \%$. Other common causes of DILI were paracetamol (14.8\%) and NSAID's (11.1\%). The most common pattern of liver injury seen was mixed pattern which was present in $63 \%$, followed by cholestatic and hepatocellular pattern. Hence, we should be very careful while prescribing these frequently used drugs.
\end{abstract}

\section{KEYWORDS}

Anti tubercular drugs, Drug Induced Liver Injury(DILI), hepatotoxicity, hepatotoxic drugs

\section{CORRESPONDING AUTHOR}

Dr. Anuj K.C.

Department of Internal Medicine,

Nepal Medical College Teaching Hospital,

Attarkhel, Gokarneshwor-8, Kathmandu Nepal

Email: anujkc11@gmail.com

ORCID ID: 0000-0001-9454-177X 


\section{INTRODUCTION}

Drug induced liver injury (DILI) is one of the common cause of liver toxicity. Most of drugs used today are hepatotoxic. It accounts for approximately one-half of the cases of acute liver failure and mimics all forms of acute and chronic liver disease. ${ }^{1}$ As liver is responsible for concentrating and metabolizing a majority of medications, it is a prime target for medicationinduced damage. It is also a common cause of acute liver failure, accounting for $13 \%$ in the United States. ${ }^{2}$ It is also the single most common adverse drug reaction leading to a halt in the development of new medications by pharmaceutical companies, failure of new drugs to obtain regulatory approval and withdrawal or restriction of existing drugs from the market. ${ }^{3-5}$ Its annual incidence in general population ranges from 14 to 19 per 100,000 inhabitants, with approximately $30 \%$ exhibiting jaundice. ${ }^{6,7}$ Overall mortality from 10 to $17.3 \%$ has been observed in several series. ${ }^{8-11}$

According to the Guidelines of American College of Gastroenterology (2014), diagnosis is made by evaluating alteration of liver enzymes, clinical history and physical examination of the patient. The criteria for DILI were those established by Indian Network for DILI (INDILI) in 2012. DILI is defined as:

1. Aspartate aminotransferase (AST) or alanine aminotransferase (ALT) levels greater than 5 times the upper limit of normal (ULN), regardless of symptoms or,

2. Total bilirubin greater than $2 \mathrm{mg} / \mathrm{dl}$ and rise in AST or ALT or alkaline phosphatase (ALP) levels greater than 2 times the ULN or,

3. AST or ALT greater than 3 times ULN, if symptomatic with nausea, vomiting, abdominal pain, anorexia, skin rash, etc.

DILI is divided into three types: hepatocellular, cholestatic and mixed according to the Councils for International Organizations of Medical Sciences (CIOMS).The pattern of liver injury is assessed by the ratio $\mathrm{R}=(\mathrm{ALT} / \mathrm{ALP})$, which allows us to define if the DILI has a hepatocellular pattern $(R>5)$, a cholestatic pattern $(\mathrm{R}<2)$ or a mixed pattern $(2<$ $\mathrm{R}<5) .{ }^{11}$ Liver injury is likely to be more severe in hepatocellular type than in cholestatic and mixed type, and patients with elevated bilirubin levels in hepatocellular liver injury indicating serious liver injury with fatalities, are found at a rate of 0.7 to $1.3 / 100,000$ individuals receiving a given drug. ${ }^{12}$

\section{MATERIALS \& METHODS}

This is a prospective cross sectional study conducted in Department of Internal Medicine of Nepal Medical College Teaching Hospital (NMCTH). Total of 27 patients of DILI, both inpatient and outpatient, were included from November 2018 to July 2019.

\section{INCLUSION CRITERIA}

All patients who present with signs and symptoms of liver toxicity and deranged liver function test according to INDILI criteria, after ingestion of any drug at least for 5 days were included in the study.

\section{EXCLUSION CRITERIA}

1. Patient taking any Ayurveda medicine

2. Alcohol dependent patient as per CAGE questionnaire

3. Patients with history of previous liver disease and/or Hepatitis B and/or C infected patients

4. Not giving consent

5. Pregnancy

6. Patients with heart failure

7. Patients with fever or any evidence of other infections

8. Ultrasonography showing abnormal liver finding e.g. fatty liver, coarse echo texture, obstructive jaundice etc.

Liver function test which included total bilirubin, direct bilirubin, AST, ALT, ALP, prothrombin time (PT), serum protein, serum albumin were done. They were all measured in Vitrous 250 machine at NMCTH. Viral markers like HBsAg, Anti HCV, IgM Anti hepatitis $A$ and $E$ virus were done to rule out any viral infections. Ultrasonogram of abdomen was done in fasting state by a radiologist to rule out any hepatobiliary obstruction.

\section{Assessment of pattern of liver injury:}

Assessing the pattern of liver injury as hepatocellular, cholestatic, or mixed was done based on calculating the " $R$ " ratio, defined by the ratio of serum ALT to ALP. The R ratio applied to each case was calculated based on the initial liver tests at presentation. The cases were classified as hepatocellular if the $\mathrm{R}$ ratio was greater than 5 , as cholestatic if the ratio was less than 2 and as mixed if the ratio was between 2-5.

Ethical clearance was taken from Institutional Review Committee (IRC) at NMCTH.Written consent was taken with each patient before the study. 
The data were collected and entered and analyzed using Epi Info version 7 to calculate mean and percentage.

\section{RESULTS}

A total of 27 patients were enrolled in the study. Out of which 14 (51.8\%) were male and 13 (48.2\%) were female. Mean age of the patients enrolled was 38.0 years. The oldest patient enrolled was 76 years of age while the youngest was 20 years of age.

Mean AST, ALT \& ALP was $328.2 \mathrm{mg} / \mathrm{dl}, 346.3$ $\mathrm{mg} / \mathrm{dl} \& 170.6 \mathrm{mg} / \mathrm{dl}$ respectively. Mean total bilirubin was $2.7 \mathrm{mg} / \mathrm{dl}$. Most common cause of DILI was anti tubercular drugs, which was the causative agent in $13(48.2 \%)$ cases. Next common causative agent was paracetamol which was responsible in $4(14.8 \%)$ cases. NSAIDs caused DILI in 3 (11.1\%) patients which included nimesulide and combination of paracetamol and ibuprofen. This was followed by antiseizure drugs especially phenytoin and sodium valproate which caused DILI in $2(7.4 \%)$ cases. One case each was consequence of antileprosy (dapsone), atorvastatin, methotrexate and warfarin. One patient had DILI but he was taking both anti tubercular therapy and phenytoin so it was difficult to distinguish the real causative agent.

Most common pattern of DILI was mixed pattern. Seventeen (63\%) cases showed mixed pattern of DILI. While $8(29.6 \%)$ cases showed cholestatic pattern and $2(7.4 \%)$ cases showed hepatocellular pattern in the study. Amongst patients who had DILI due to ATT, 7 (53.8\%) had mixed pattern.

\section{DISCUSSION}

The most common cause of DILI in our study was anti tubercular therapy. In our study, $48.2 \%$ of the cases of DILI were due to ATT drugs which is almost similar to the findings done in Mumbai by Rathi C et al, ${ }^{13}$ which had $49 \%$. This similar result

\begin{tabular}{|lccc|}
\multicolumn{4}{c}{ Table 1: Pattern of DILI } \\
Drugs & Mixed & $\begin{array}{c}\text { Pattern } \\
\text { Cholestatic }\end{array}$ & Hepatocellular \\
Antitubercular Drugs & 7 & 5 & 1 \\
Paracetamol & 3 & 1 & 0 \\
NSAIDS & 2 & 0 & 1 \\
Antiseizure & 2 & 0 & 0 \\
Antileprosy & 1 & 0 & 0 \\
Atorvastatin & 0 & 1 & 0 \\
Warfarin & 0 & 1 & 0 \\
Methotrexate & 1 & 0 & 0 \\
ATT + Phenytoin & 1 & 0 & 0 \\
Total & $\mathbf{1 7}$ & $\mathbf{8}$ & 2 \\
\hline
\end{tabular}

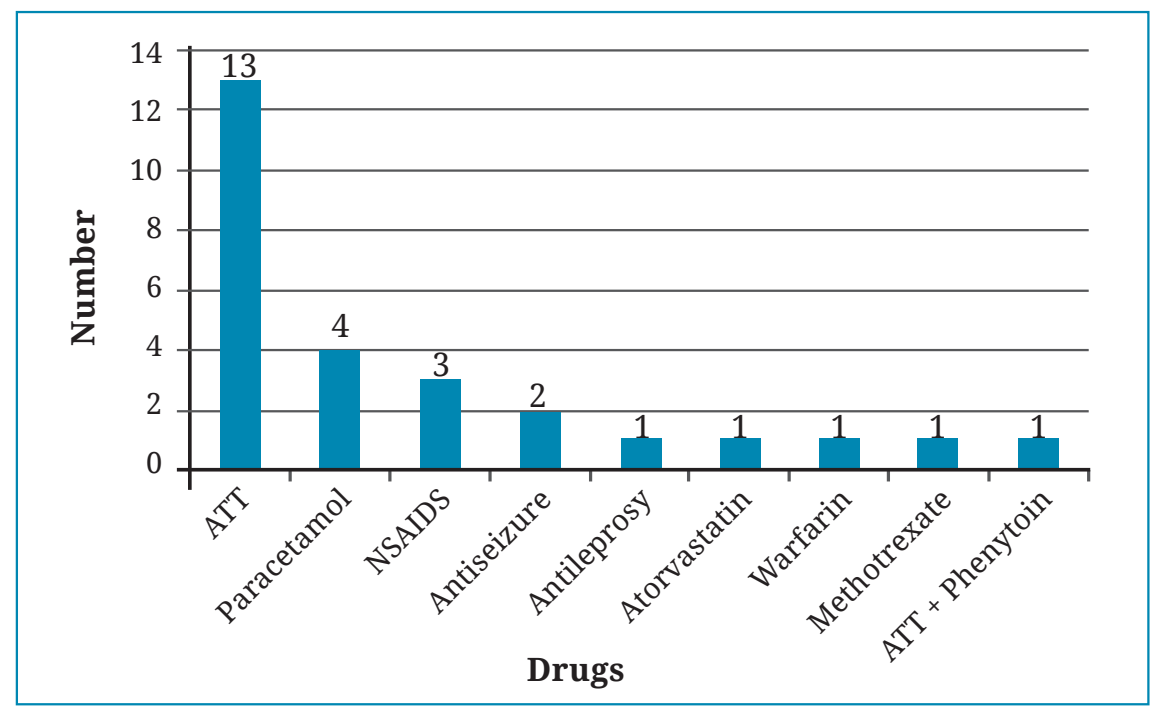

Fig. 2: Etiology of DILI

(ATT: Antitubercular drugs; NSAIDs: Nonsteroidal anti-inflammatory drugs) 
was probably because tuberculosis is one of the most common causes of infection in our part of subcontinent. Another study also supported our study which was done by Treeprasertsuk $S$ et $a l^{14}$ in Bangkok which showed $85 \%$ of DILI was due to ATT. In another study by Shen T et $a l,{ }^{15}$ in China, ATT (21.99\%) was second only to traditional chinese herbal medicine as a common cause of DILI. These suggest ATT is quite common cause of DILI. Next in line was paracetamol and NSAIDs which was the causative agent in $14.8 \%$ and $11.1 \%$ respectively. Paracetamol overdose is commonly encountered case in our settings as it is one of the frequently used over the counter drug along with NSAIDs. Rathi et $a l^{13}$ had also similar causative agents as our study after ATT, it was antiepileptic, NSAID's, methotrexate, atorvastatin and antileprosy.

In our study most common pattern of DILI was mixed pattern (63\%), followed by cholestatic pattern (29.6\%) and hepatocellular pattern (7.4\%) which is in contrast to the study done by Licata et al. ${ }^{16}$ In their study, hepatocellular pattern was commonest (57.8\%) followed by cholestatic (18.3\%) and mixed pattern (23.2\%). This contrasting result probably could be due to the difference in drugs which led to DILI in the study, as most common cause was due to NSAIDs followed by antibiotics excluding antitubercular therapy. In another study by Rathi $\mathrm{C}$ et $a l,{ }^{13}$ hepatocellular pattern was most common (50\%) followed by mixed pattern (35\%) and cholestatic pattern(15\%). In our study ATT was the most common cause of DILI which also showed mixed pattern (53.8\%) as most common pattern of presentation.

Drug induced liver injury may be quite common in our part of the world especially with these drugs which we are using frequently. We miss this frequently because we send the investigations only after patients develop symptoms. Though Acute Hepatic Failure is less common with drugs but few cases have been reported. So we should be very careful, before prescribing these frequently used drugs.

\section{REFERENCES}

1. Kaplowitz N. Drug-induced liver disorders: implications for drug development and regulation. Drug Saf 2001; 24: 483-90.

2. Ostapowicz G, Fontana RJ, Schiødt FV, et al. U.S. Acute Liver Failure Study Group. Results of a prospective study of acute liver failure at 17 tertiary care centers in the United States. Ann Intern Med 2002; 137: 947-54.

3. Watkins PB, Seeff LB. Drug-induced liver injury: summary of a single topic clinical research conference. Hepatol 2006; 43: 618-31.

4. Navarro VJ, Senior JR. Drug-related hepatotoxicity. N Engl J Med 2006; 354: 731-9.

5. Abboud G, Kaplowitz N. Drug-induced liver injury. Drug Saf 2007; 30: 277-94.

6. Bjornsson E, Bergmann OM, Bjornsson HK, Kvaran $\mathrm{RB}$, Olafsson S. Incidence, presentation, and outcomes in patients with drug induced liver injury in the general population of Iceland. Gastroenterol 2013; 144: 1419-25.

7. Sgro C, Clinard F, Ouazir K et al. Incidence of drug induced hepatic injuries: a French populationbased study. Hepatol 2002; 36: 451-5.

8. Chalasani N, Bonkovsky HL, Fontana R et al. Features and outcomes of 899 patients with druginduced liver injury: The DILIN prospective study. Gastroenterol 2015; 148: 1340-52.

9. Devarbhavi H, Dierkhising R, Kremers WK, Sandeep MS, Karanth D, Adarsh CK. Single-center experience with drug-induced liver injury from
India: causes, outcome, prognosis, and predictors of mortality. Am J Gastroenterol 2010; 105: 2396404.

10. de Abajo FJ, Montero D, Madurga M, García Rodríguez LA. Acute and clinically relevant druginduced liver injury: a population based casecontrol study. Br J Clin Pharmacol 2004; 58: 71-80.

11. Chalasani NP, Hayashi PH, Bonkovsky HL, Navarro VJ, Lee WM, Fontana RJ. ACG Clinical Guideline: the Diagnosis and management of idiosyncratic drug induced liver injury. Am J Gastroenterol 2014; 109: 950-6.

12. Nepal National TB Programme, Annual Report 2010/2011

13. Rathi C, Pipaliya N et al. Drug Induced Liver Injury at a Tertiary Hospital in India: Etiology, Clinical Features and Predictors of Mortality. Ann Hepatol 2017; 16: 442-50.

14. Treeprasertsuk S, Huntrakul J, Ridtitid W, Kullavanijaya P, Björnsson ES. The predictors of complications in patients with drug-induced liver injury caused by antimicrobial agents. Aliment Pharmacol Ther 2010; 31: 1200-7.

15. Shen T, Liu Y, Shang J et al. Incedence \& etiology of drug induced liver injury in mainland China. Gastroenterol 2019; 156: 2230-41.

16. Licata A, Minissale MG, Calvaruso V, Craxi A. A focus on epidemiology of drug-induced liver injury: analysis of a prospective cohort. Eur Rev Med Pharmacol Sci 2017; 21: 112-21. 